\title{
Zwint facilitates melanoma progression by promoting c-Myc expression
}

\author{
KUANHOU MOU, JIAN ZHANG, XIN MU, LIJUAN WANG, WENLI LIU and RUI GE
}

\begin{abstract}
Department of Dermatology, The First Affiliated Hospital of Xi'an Jiaotong University, Xi'an, Shaanxi 710061, P.R. China
\end{abstract}
Received November 6, 2020; Accepted April 26, 2021

DOI: $10.3892 / \mathrm{etm} .2021 .10250$

\begin{abstract}
ZW10 interactor (Zwint) is upregulated in various types of tumors and exerts a carcinogenic effect. However, little is known about the expression profile, function and molecular mechanisms of action of Zwint in melanoma. Therefore, the aim of the present study was to investigate the expression levels of Zwint in melanoma cell lines and tissues. It was revealed that Zwint was highly expressed in melanoma samples. Functional experiments indicated that Zwint knockdown suppressed the proliferation and migration of A375 melanoma cells. Further mechanistic studies demonstrated that Zwint knockdown decreased the protein expression levels of c-Myc, MMP-2, Slug, mTOR, phosphorylated (p)-mTOR, p-p38 and fibronectin, while it increased the protein expression levels of E-cadherin and MMP-9. Among these genes, c-Myc, MMP-2 and Slug were overexpressed to investigate their effects on cell proliferation following Zwint knockdown. The results demonstrated that overexpression of c-Myc, but not MMP-2 or Slug, rescued the effects of Zwint knockdown on melanoma cell proliferation and migration. Taken together, the results of the present study suggested that Zwint may act as an oncogene in melanoma by regulating c-Myc expression.
\end{abstract}

\section{Introduction}

Melanoma, which is derived from melanocytes, is the most aggressive type of skin cancer (1). According to epidemiological studies, the incidence and mortality rates of patients with melanoma are continuing to increase in the United States (2). Despite improvements in the diagnosis and treatment of melanoma, the prognosis of patients with advanced melanoma remains unfavorable, with a 3 -year survival rate of only $15 \%(3,4)$. Therefore, it is critical to define the molecular

Correspondence to: Dr Rui Ge, Department of Dermatology, The First Affiliated Hospital of Xi'an Jiaotong University, 277 Yanta West Road, Xi'an, Shaanxi 710061, P.R. China

E-mail: green-0074@163.com

Key words: melanoma, ZW10 interactor, c-Myc, proliferation, migration mechanisms of action underlying melanoma growth and metastasis, with the aim to develop novel therapeutic strategies.

ZW10 interactor (Zwint) is a kinetochore protein found in higher eukaryotes, which plays an important role in the mitotic checkpoint $(5,6)$. The interaction between Zwint and ZW10 mediates stable ZW10 kinetochore residency at prometaphase kinetochores, which is indispensable for mitotic checkpoint arrest $(7,8)$. Given that the mitotic checkpoint is a primary mechanism ensuring high fidelity of segregation during mitosis, abnormalities often lead to the development of tumors (9). Therefore, it may be hypothesized that an abnormal expression or structure of Zwint may be associated with carcinogenesis. Previous studies have reported that Zwint is overexpressed in various types of tumors and is associated with a poor prognosis (10-12). In addition, it has been demonstrated that Zwint may act as an oncogene $(11,13)$. However, whether Zwint is associated with melanoma progression and its specific molecular mechanism of action remain unclear.

The aim of the present study was to investigate whether Zwint regulates melanoma growth and metastasis and whether its effects are mediated by promoting c-Myc expression, in order to determine whether Zwint may serve as a novel target for the treatment of melanoma.

\section{Materials and methods}

Data Download. Microarray data set (GSE46517) (14) was downloaded from Gene Expression Omnibus (ncbi.nlm.nih. gov/geo/query/acc.cgi?acc=GSE46517). The expressing level of Zwint in 31 primary melanoma samples and 9 nevi tissues were from GSE46517 (GPL96 Affymetrix Human Genome U133A Array).

Cell culture. The human melanoma cell lines A375, A2058 (amelanotic melanoma) and 451Lu (metastatic melanoma) were purchased from GeneChem, Inc. A375 and A2058 were maintained in DMEM (Gibco; Thermo Fisher Scientific, Inc.) supplemented with $10 \%$ FBS (Invitrogen; Thermo Fisher Scientific, Inc.). 451Lu cell lines were cultured in RPMI-1640 medium (Invitrogen; Thermo Fisher Scientific, Inc.) supplemented with 10\% FBS (Invitrogen; Thermo Fisher Scientific, Inc.). The immortalized normal human epidermal melanocyte cell line, PIG1 (a gift from Dr Caroline Le Poole, Loyola University Chicago, USA), was cultured in Medium 254 (Invitrogen; Thermo Fisher Scientific, Inc.) supplemented with 
Human Melanocyte Growth Supplement (Invitrogen; Thermo Fisher Scientific, Inc.) and 5\% FBS (Invitrogen; Thermo Fisher Scientific, Inc.).

Plasmids, short hairpin (sh)RNA and transfection. The lenti-plasmid vector system CV224 (Ubi-MCS-SV40-Cherry-IRES-puromycin) and lenti-shRNA vector system GV115 (hU6-MCS-CMV-EGFP) were constructed, packed and purified by GeneChem, Inc. A second generation system was used for lentivirus construction. Zwint targeting shRNA-1 (5'-CCGGCAGAGAATCTTCCAGAT GATACTCGAGTATCATCTGGA AGATTCTCTGTTT TTG-3') or shRNA-2 (5'-CCGGCTACAACCTGCTGGAGA TGTACTCGAGTACATCTCCAGCAGGTTGTAGTTTTT G-3') and control shRNA (5'-CCGGCCTTCTCCGAACGT GTCACGTCTCGAGTAAATGCTGGTACTCAAATGGTT TTT-3') were designed with a hairpin and inserted into the GV115 vector. c-Myc cDNA, MMP-2 cDNA and Slug cDNA were cloned into CV224 vector and empty vector was used as negative control. Then, 293T cells (Cell Bank of the Chinese Academy of Sciences) were seeded into a $10 \mathrm{~cm}$ dish $\left(5 \times 10^{6}\right.$ cells) and transfected using Lipofectamine ${ }^{\circledR} 3000$ (Invitrogen; Thermo Fisher Scientific, Inc.) according to the manufacturer's protocol. The vectors were transfected in the following proportions: $\mathrm{GV} / \mathrm{CV}$ vector plasmid: pHelper1.0 vector plasmid: Phelper 2.0 vector plasmid; $20 \mu \mathrm{g}$ : $15 \mu \mathrm{g}$ : $10 \mu \mathrm{g}$ for $48 \mathrm{~h}$ at $37^{\circ} \mathrm{C}$ in an incubator before the $293 \mathrm{~T}$ lentiviral supernatant was harvested by centrifugation at $4,000 \mathrm{x}$ g for $10 \mathrm{~min}$ at room temperature. Phelper 1.0 vector contains the gag gene of HIV virus, encoding the main structural proteins of the virus; the pol gene, encoding a virus-specific enzyme; the rev gene encodes regulatory factors that regulate the expression of gag and pol genes. Phelper 2.0 vector contains the herpes simplex virus-derived VSV-G gene, which provides the envelope protein needed for virus packaging. The lentiviral supernatant was concentrated and purified for further concentration detection, and 10 MOI was used for the next transfection. A375 cells were seeded into a six-well plate at a density of $1 \times 10^{5}$ cells/well and were infected with the lenti-shRNA vectors alone or co-infected with lenti-plasmid-Cherry vectors for $24 \mathrm{~h}$. After that, culture medium containing the virus was replaced with fresh culture medium. Cells were collected $72 \mathrm{~h}$ after infection for further analysis. The expression levels of the reporter gene Cherry or GFP were observed using fluorescence microscopy (Olympus Corporation; magnification, $\mathrm{x} 100)$. If the fluorescence rate was $>70 \%$ and the cells were considered healthy, subsequent experiments were performed.

Reverse transcription-quantitative (RT-q)PCR. Total RNA extracted from the melanoma cells was isolated using TRIzol ${ }^{\circledR}$ reagent (Invitrogen; Thermo Fisher Scientific, Inc.) and then reverse transcribed with Superscript II Reverse Transcriptase (Invitrogen; Thermo Fisher Scientific, Inc.) according to the manufacturer's protocol. RT-qPCR experiments were performed using the SYBR-Green Premix Ex Taq kit (Takara Biotechnology, Co., Ltd.) according to the manufacturer's protocol. BIO-RAD MJ Mini Opticon Real-Time PCR System was used to determine the relative mRNA levels. The following thermocycling conditions were used: Initial denaturation at $95^{\circ} \mathrm{C}$ for $30 \mathrm{sec}, 40$ cycles at $95^{\circ} \mathrm{C}$ for $5 \mathrm{sec}, 60^{\circ} \mathrm{C}$ for $30 \mathrm{sec}$ and $72^{\circ} \mathrm{C}$ for $20 \mathrm{sec}$. Threshold cycle values were used to calculate the fold-change in the transcript levels by using the $2^{-\Delta \Delta C q}$ method (15). The relative mRNA expression levels were normalized to the GAPDH gene. The following primer sequences were used: Zwint forward, 5'-CACGTAGAGGCC ATCAAAATTGG-3' and reverse, 5'-CGGAGTTGTGTCCGT TTCCT-3; GAPDH forward, 5'-TGACTTCAACAGCGACAC CCA-3' and reverse, 5'-CACCCTGTTGCTGTAGCCAAA-3'; MMP-2 forward, 5'-GATACCCCTTTGACGGTAAGGA-3' and reverse, 5'-CCTTCTCCCAAGGTCCATAGC-3'; Slug forward, 5'-GCCAAACTACAGCGAACTGG-3' and reverse, 5'-ATTGGGTAGCTGGGCGTGGA-3'; c-Myc forward, 5'-TGGTGCTCCATGAGGAGACA-3'; and reverse, 5'-TGT GAGGAGGTTTGCTGTGG-3'.

Western blot analysis. Western blot analysis was performed as previously described (16). Melanoma cells were washed with phosphate buffered saline (PBS) and lysed using RIPA lysis buffer (Xi'an Runde Biotechnology Co., Ltd.) and phenylmethanesulfonyl fluoride (protease inhibitor mix; Sigma-Aldrich; Merck KGaA). Cell lysates were cleared by centrifugation for $15 \mathrm{~min}$ at $13,000 \mathrm{x} \mathrm{g}$ at $4^{\circ} \mathrm{C}$. Total protein was quantified using a bicinchoninic acid assay kit (Beyotime Institute of Biotechnology) and $20 \mu \mathrm{g}$ protein/lane was separated by $10 \%$ SDS-PAGE and blotted onto a nitrocellulose membrane. Following blocking in a solution of 5\% non-fat dry milk diluted in tris-buffered saline (TBS; pH 7.4) containing $0.05 \%$ Tween-20 at room temperature for $1 \mathrm{~h}$, the membranes were washed and incubated with primary antibodies overnight at $4^{\circ} \mathrm{C}$. After washing, the membranes were incubated with horseradish peroxidase-conjugated secondary antibodies for $1 \mathrm{~h}$ at room temperature. Bound antibodies were detected using a chemiluminescence detection kit (KPL, Inc.). Primary antibodies against Zwint (1:1,000; cat. no. ab71982), c-Myc (1:1,000; cat. no. ab32072), Twist (1:1,000; cat. no. ab50887), p-mTOR (1:2,000; cat. no. ab109268), N-Cadherin (1:1,000; cat. no. ab18203), Fibronectin (1:500; cat. no. ab32419), and GAPDH (1:2,000; cat. no. ab37168) were purchased from Abcam. Antibodies against Slug (1:1,000; cat. no. 9585), MMP-2 (1:500; cat. no. 13132), p-AKT (1:1,000; cat. no. 4060), Snail (1:2,000; cat. no. 3879), AKT $(1: 2,000$; cat. no. 4691), $\beta$-catenin (1:2,000; cat. no. 8480), NF-кB p65 (1:2,000; cat. no. 8242), Vimentin (1:1,000; cat. no.3932), p-NF-кB p65 (1:1,000; cat. no. 3033), p-p38 (1:1,000; cat. no. 4631), MMP-9 (1:500; cat. no. 13667), p38 (1:2,000; cat. no. 8690), p- $\beta$-catenin (1:1,000; cat. no. 2009), mTOR (1:500; cat. no. 2972), ERK $(1: 2,000$; cat. no. 9102), E-Cadherin (1:2,000; cat. no. 9107) and p-ERK (1:2,000; cat. no. 4376) were purchased from Cell Signaling Technology, Inc. Secondary antibodies including anti-rabbit $\operatorname{IgG}(1: 10,000$; cat. no. AS1107) or anti-mouse IgG (1:10,000; cat. no. AS1106) were purchased from ASPEN Biotechnology CO., LTD.

Cell proliferation assay. Following transduction, A375 cells from each group were trypsinized and counted. Cells were seeded into 96 -well plates at a density of $2 \times 10^{3}$ cells/well in quintuplicate and incubated at $37^{\circ} \mathrm{C}$ for $1,2,3,4$ and 5 days. Cell numbers were counted over the 5 continuous days. 

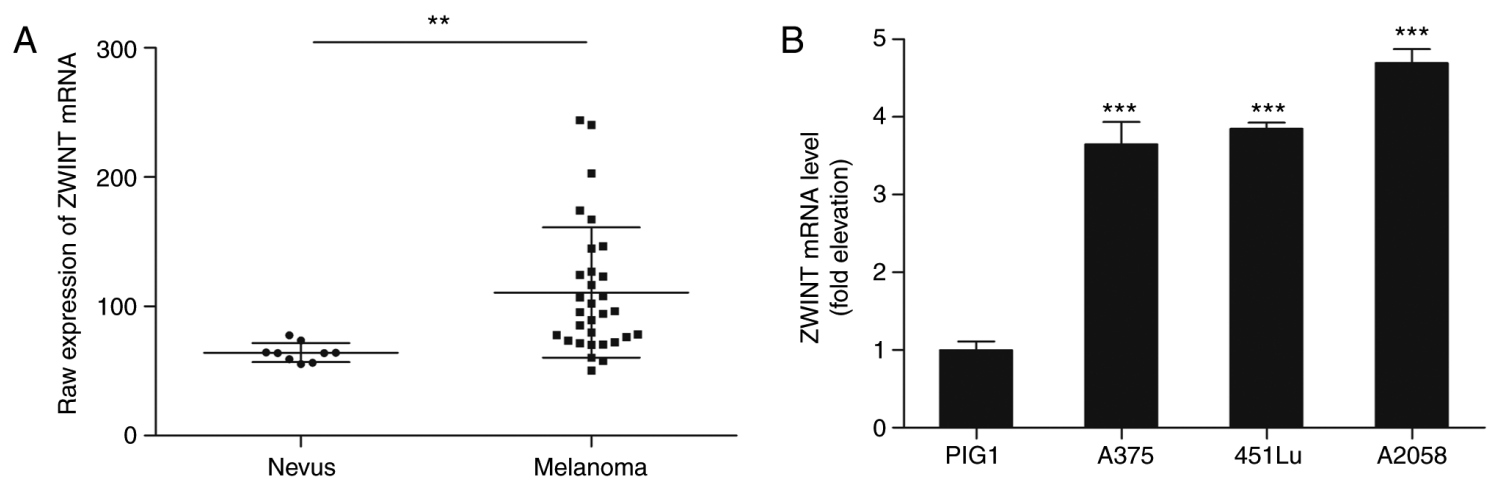

Figure 1. Zwint mRNA is highly expressed in melanoma tissues and cells. (A) Zwint mRNA expression was upregulated in melanoma tissues (n=31) compared with nevus tissues ( $n=9$ ), according to the GSE46517 dataset. (B) Reverse transcription-quantitative PCR analysis in different melanoma cell lines indicated that Zwint expression levels were increased in melanoma cells compared with PIG1 normal human epidermal melanocytes. ${ }^{* *} \mathrm{P}<0.01$ vs. nevus; ${ }^{* * * *} \mathrm{P}<0.001 \mathrm{vs}$. PIG1. ZWINT, ZW10-interactor.

Fluorescence microscopy was used to assess the cell counts using Celigo Imaging Cytometer (Nexcelom Bioscience LLC; magnification, x100). Cell proliferation was also assessed using MTT assay (Beijing Dingguo Changsheng Biotechnology Co., Ltd.). Following transduction, cells were incubated with $20 \mu \mathrm{l} \mathrm{MTT}$ at $37^{\circ} \mathrm{C}$ for $4 \mathrm{~h}$. Following MTT incubation, the purple formazan crystals were dissolved using DMSO and cell proliferation was subsequently analyzed at a wavelength of $490 \mathrm{~nm}$ using a microplate reader (infinite M2009PR; Tecan Group, Ltd.).

Transwell assay. Following transduction, A375 cells were trypsinized and resuspended in serum-free DMEM at a density of $1 \times 10^{5}$ cells $/ \mathrm{ml}$. Cell suspensions $(100 \mu \mathrm{l})$ were plated in the upper chambers (pore size, $8 \mu \mathrm{m}$; Corning, Inc.), while $600 \mu 1$ DMEM supplemented with $30 \%$ FBS was plated in the lower chambers. Following incubation at $37^{\circ} \mathrm{C}$ for $24 \mathrm{~h}$, the migratory cells were fixed with $4 \%$ paraformaldehyde for $30 \mathrm{~min}$ at room temperature and subsequently stained with $4 \mathrm{~g} / \mathrm{l}$ crystal violet for $10 \mathrm{~min}$ at room temperature. Stained cells were counted in nine randomly selected fields using a light microscope (Olympus Corporation; magnification, x100).

Statistical analysis. Statistical analysis was performed using GraphPad Prism v5.00 software (GraphPad Software, Inc.). All experiments were performed in triplicate and data are presented as the mean $\pm \mathrm{SD}$. Unpaired student's t-test was used to compare differences between two groups. One-way ANOVA followed by Tukey's post-hoc test was used for multiple comparisons. $\mathrm{P}<0.05$ was considered to indicate a statistically significant difference.

\section{Results}

Zwint expression in melanoma. To determine the role of Zwint in melanoma, the GSE46517 dataset was downloaded from the Gene Expression Omnibus database. The analysis demonstrated that Zwint expression was significantly upregulated in primary melanoma lesions compared with nevus samples $(\mathrm{P}=0.00178$; Fig. 1A). Subsequently, the mRNA level of Zwint was examined in various melanoma cell lines $(\mathrm{A} 375,451 \mathrm{Lu}$ and A2058) as well as in the immortalized normal human epidermal melanocyte cell line PIG1. The mRNA level of Zwint in all melanoma cell lines was increased compared with that in the PIG1 cells (Fig. 1B).

Zwint knockdown suppresses the proliferation and migration of melanoma cells. To investigate the effect of Zwint knockdown on the malignant growth potential, A375 cells were transfected with two different shRNAs targeting Zwint. Western blot and RT-qPCR analyses demonstrated that Zwint expression significantly decreased following transfection with both shRNAs compared with the control shRNA (Fig. 2A and B). The proliferation of melanoma cells was assessed via cell counting and MTT assay. The results demonstrated that Zwint knockdown notably decreased cell proliferation (Fig. 2C and D). The ability of Zwint to modify the motility of melanoma cells was also assessed. The results of the Transwell migration assay demonstrated that A375 cells transfected with either Zwint shRNA-1 or shRNA-2 migrated at a slower rate compared with the control cells (Fig. 2E). Notably, the inhibitory effect on cell proliferation and migration was more pronounced in A375 cells transfected with Zwint shRNA-1 compared with Zwint shRNA-2. Thus, Zwint shRNA-1 was selected for subsequent experiments.

Rescue of c-Myc in Zwint knocked-down cells restores cell proliferation and migration. Given the notable effects of Zwint knockdown on melanoma cells, the signaling pathways involved in tumor growth and migration were assessed via western blot analysis. The results demonstrated that the expression levels of c-Myc, MMP-2, Slug, mTOR, phosphorylated (p)-mTOR, p-p38 and fibronectin were decreased following Zwint knockdown, while the expression levels of E-cadherin and MMP-9 were increased. However, the expression levels of ERK, p-ERK, N-cadherin, Snail, AKT, p-AKT, $\beta$-catenin, $p$ - $\beta$-catenin, p38, NF- $\kappa B$ p65, p- NF- $\kappa B$ p65, Twist and Vimentin did not change following Zwint knockdown. (Fig. 3A-H). To identify which gene may serve a crucial role in mediating the function of Zwint, MMP-2, Slug and c-Myc were overexpressed to investigate their effects on cell proliferation and migration following Zwint knockdown. The overexpression efficiency of MMP-2, Slug and c-Myc vectors was verified by western blotting (Fig. S1). Furthermore, 


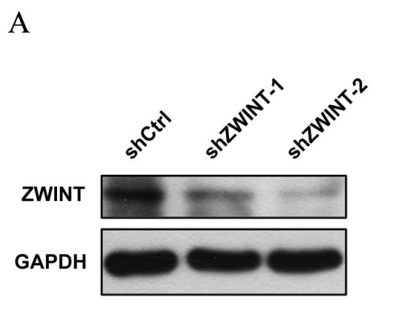

B

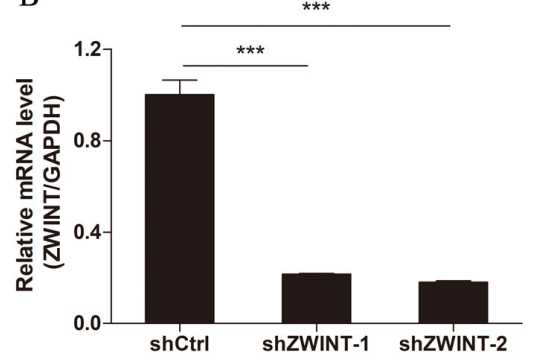

D
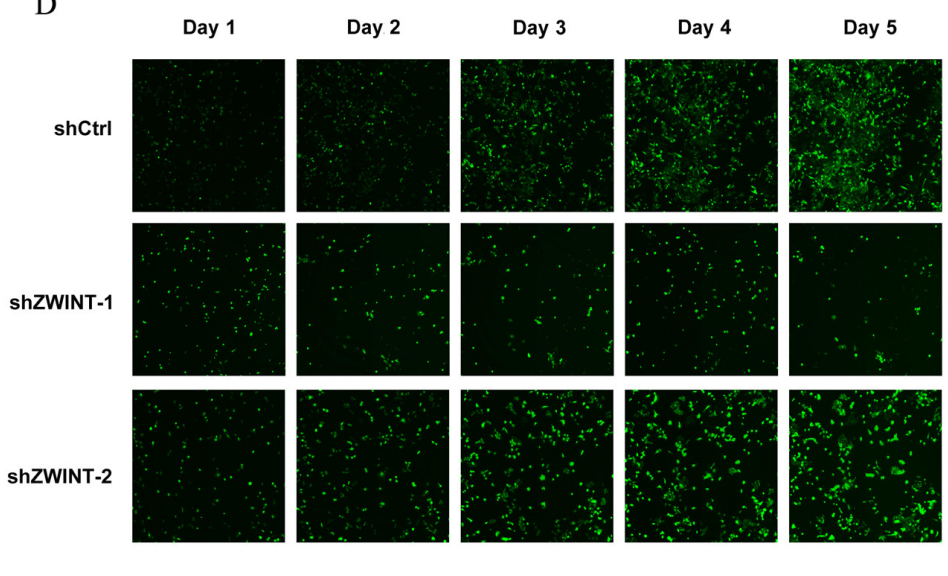

E
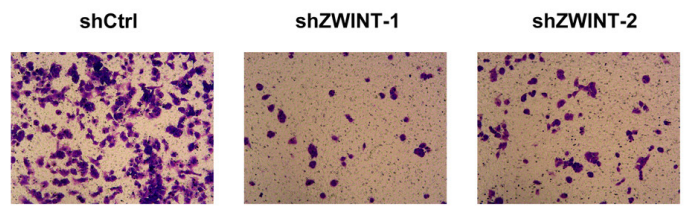

$\mathrm{C}$
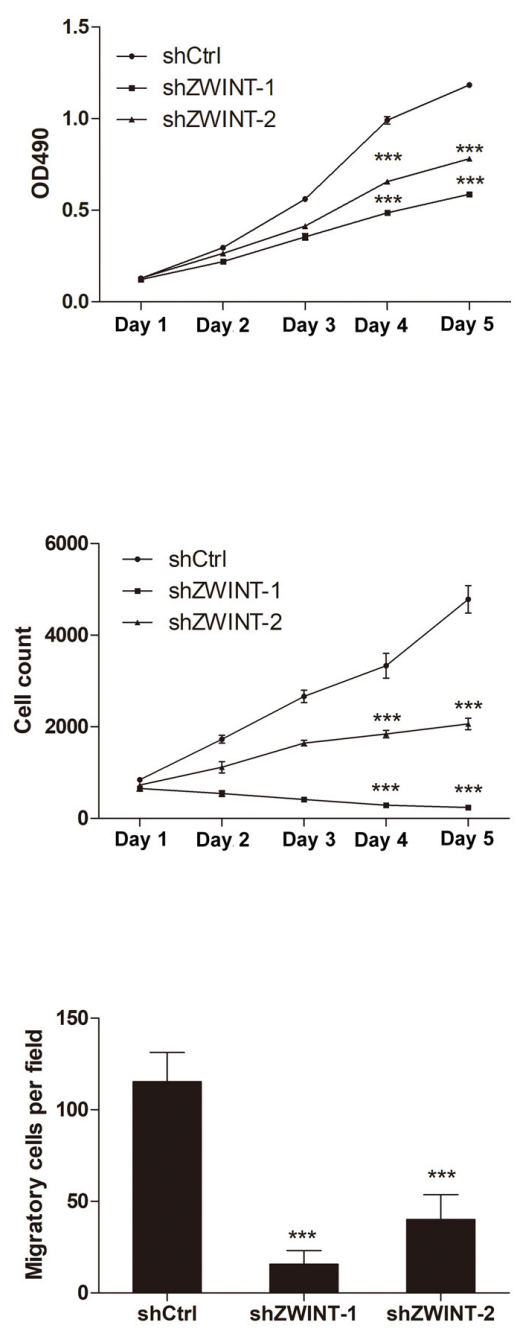

Figure 2. Zwint knockdown suppresses the proliferative and migratory ability of A375 melanoma cells. (A) Western blotting and (B) reverse transcription-quantitative PCR analyses were performed to detect the knockdown efficiency of two different shRNAs against Zwint. (C) MTT assay indicated that knockdown of Zwint inhibited A375 cell proliferation. (D) Control or Zwint knocked-down A375 cells expressing green fluorescent protein were visualized to perform cell counts using a Celigo Imaging Cytometer (magnification, x100). (E) Transwell assay demonstrated that knockdown of Zwint significantly inhibited the migration of A375 cells (magnification, $\mathrm{x} 100$ ). ${ }^{* * * *} \mathrm{P}<0.001$ vs. shCtrl. OD, optical density; sh; short hairpin; Ctrl, control; ZWINT, ZW10-interactor.
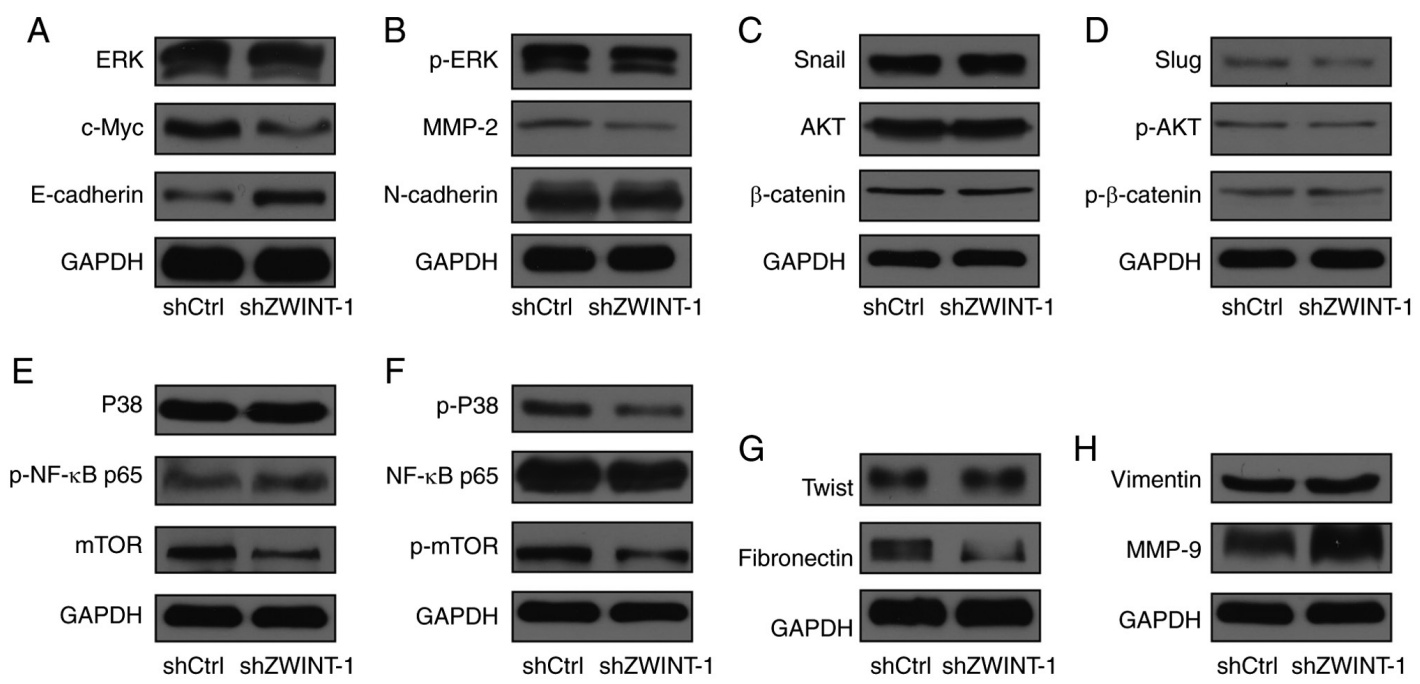

Figure 3. Screening for the downstream effectors regulated by Zwint. (A-H) Western blot analysis was performed to detect the protein expression changes of (A) ERK, c-Myc and E-cadherin; (B) p-ERK, MMP-2 and N-cadherin; (C) Snail, AKT and $\beta$-catenin; (D) Slug, p-AKT and p- $\beta$-catenin; (E) p38, p-NF- $\mathrm{kB}$ and mTOR; (F) p-p38, NF-kB and p-mTOR; (G) Twist and fibronectin; and (H) vimentin and MMP-9, following Zwint knockdown in A375 melanoma cells. p, phosphorylated; sh, short hairpin; Ctrl, control; ZWINT, ZW10-interactor. 
A

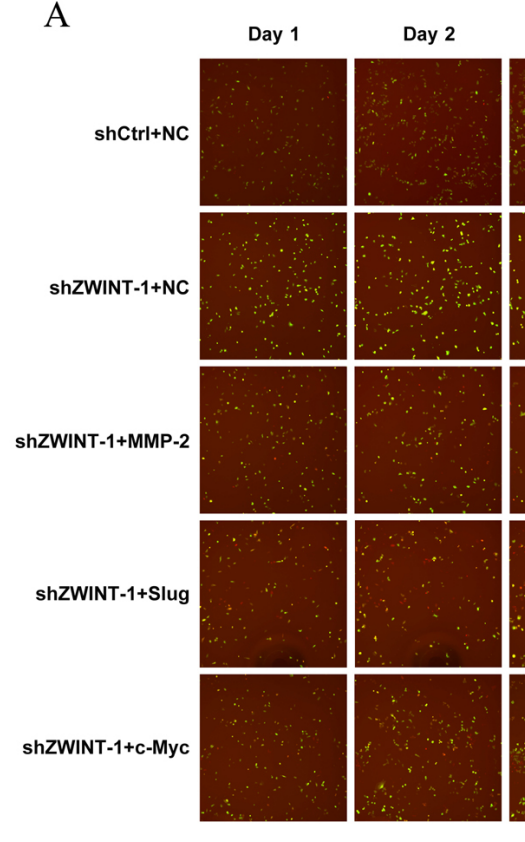

B

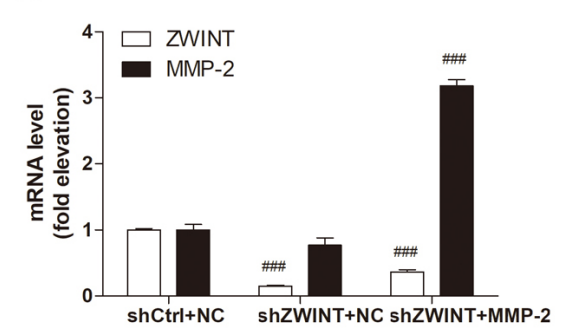

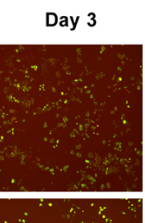
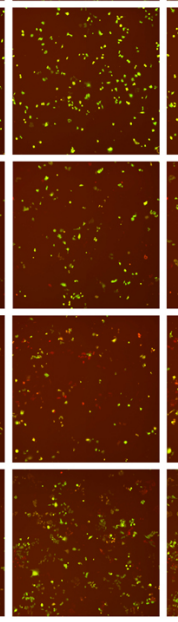

$\mathrm{C}$

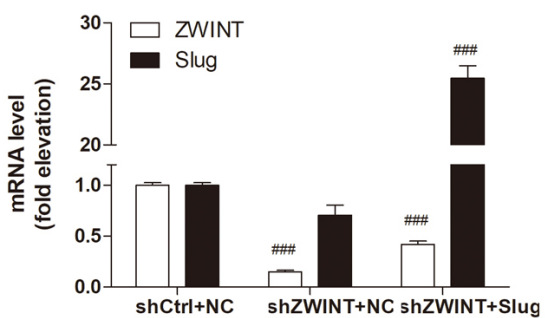

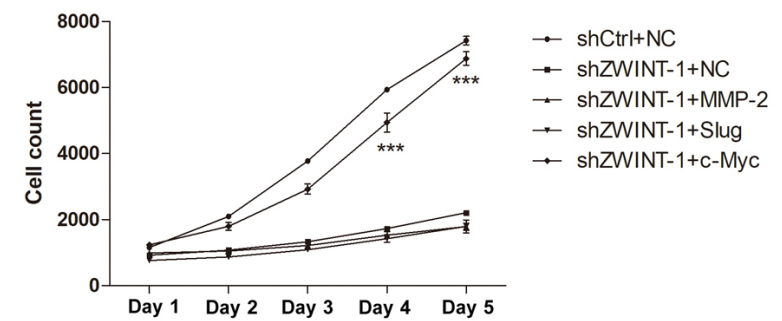

D

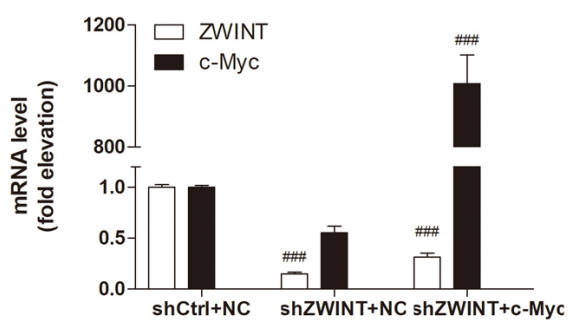

$\mathrm{E}$

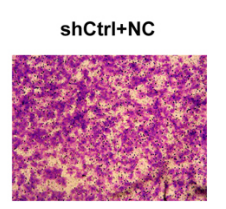

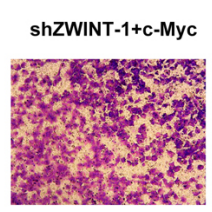

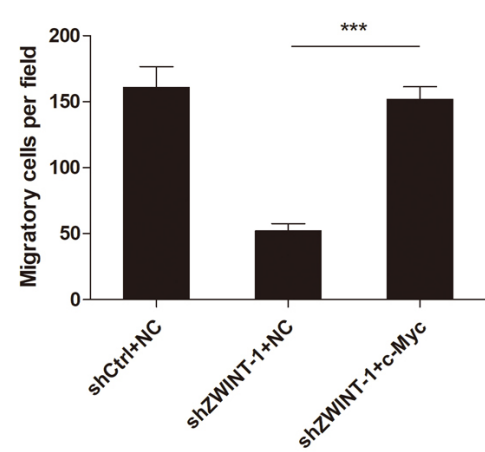

$\mathrm{F}$

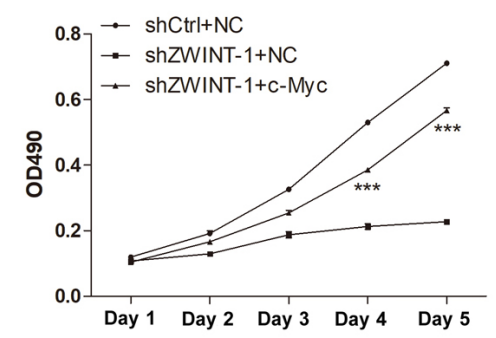

Figure 4. Rescue of c-Myc expression in Zwint depleted cells restores the proliferative and migratory abilities of A375 melanoma cells. (A) Celigo Imaging Cytometer was used to perform cell counts via fluorescence measurement in the indicated groups. Co-transfection of GFP-tagged shCtrl with Cherry-tagged NC, GFP-tagged shZWINT-1 with Cherry-tagged NC or Cherry-tagged MMP-2 or Cherry-tagged Slug or Cherry-tagged c-Myc in A375 cells (magnification, x100). (B-D) Reverse transcription-quantitative PCR analyses were performed to detect interference and overexpression efficiency following transfection with Zwint shRNAs and (B) MMP-2, (C) Slug or (D) c-Myc plasmids. (E) Transwell assay was performed to assess cell migration (magnification, x100). (F) MTT assay was performed to assess cell proliferation. ${ }^{* * *} \mathrm{P}<0.001$ vs. shZWINT-1 + NC. ${ }^{\# \#} \mathrm{P}<0.001$ vs. shCtrl + NC. GFP, green fluorescent protein; NC, negative control; sh; short hairpin; Ctrl, control; ZWINT, ZW10-interactor.

Zwint knockdown or MMP-2, Slug and c-Myc overexpression efficiency was verified by RT-qPCR (Fig. 4B-D). The results of the cell counting tests demonstrated that c-Myc overexpression promoted cell proliferation in Zwint depleted cells compared with cells transfected with the empty vector (Fig. 4A), suggesting that c-Myc may be a downstream effector of Zwint. Furthermore, the results of the MTT and Transwell migration assays demonstrated that c-Myc overexpression in
Zwint depleted cells restored melanoma cell migration and proliferation (Fig. 4E and F).

\section{Discussion}

The present study aimed to investigate the expression and function of Zwint in melanoma and elucidate its molecular mechanism. The results demonstrated that Zwint was highly 
expressed in melanoma tissues and cells. In addition, Zwint depletion suppressed melanoma cell proliferation and migration. Consistent with these results, previous studies have demonstrated that Zwint is highly expressed in different types of cancer and exerts an oncogenic effect. For example, Zhou et al (17) reported that Zwint is upregulated in breast cancer tissues compared with adjacent normal tissue and can promote breast cancer cell proliferation. Furthermore, Peng et al (13) demonstrated that Zwint is overexpressed in lung adenocarcinoma compared with adjacent normal tissue, and Zwint knockdown suppresses tumor malignant behaviors, such as proliferation, migration, invasion and colony formation. Upregulated Zwint expression has also been observed in human hepatocellular carcinoma, ovarian cancer and breast cancer compared with normal adjacent tissue and has been associated with a poor prognosis $(11,12,17)$. Consistent with previous findings, the results of the present study suggested that Zwint acts as an oncogene and may be a potential therapeutic target in melanoma. However, the molecular mechanism underlying its carcinogenic effects remains to be fully elucidated.

To further investigate the molecular mechanism of Zwint in melanoma, western blot analysis was performed to screen downstream molecules associated with cell proliferation and migration, following Zwint knockdown. These downstream candidates included key molecules in proliferation-associated MAPK and PI3K-AKT signaling pathways $(18,19)$, such as ERK, p-ERK, p38, p-p38, c-Myc, AKT, p-AKT, NF-кB $\mathrm{p} 65, \mathrm{p}-\mathrm{NF}-\kappa \mathrm{B}$ p65, mTOR and $\mathrm{p}$-mTOR. The candidates also included molecules associated with cell adhesion and extracellular matrix that promote tumor migration (20) such as Twist, Fibronectin, Snail, $\beta$-catenin, $p-\beta$-catenin, Slug, Vimentin, MMP-2, MMP-9, E-Cadherin and N-Cadherin. The results demonstrated that the expression levels of c-Myc, MMP-2, Slug, mTOR, p-mTOR, p-p38 and fibronectin were decreased following Zwint knockdown, while the expression levels of E-cadherin and MMP-9 were increased. Furthermore, subsequent experiments indicated that overexpression of c-Myc rescued the effects of Zwint depletion on melanoma cell proliferation and migration. Taken together, these results suggested that Zwint acts as an oncogene in melanoma by regulating c-Myc expression. However, whether other molecules regulated by Zwint also play a role in this process remains to be investigated. Zwint serves an important role in the spindle assembly checkpoint $(7,8)$, which suggests that it may play a role in the pathogenesis of tumors. In addition to its role in the mitotic spindle checkpoint, increasing evidence has suggested that Zwint may participate in other signaling pathways. For instance, Zwint expression was indicated to be associated with the expression levels of PCNA, Cdc25C, cyclin B1 and CDK1 (11). Li et al (10) reported that high Zwint expression was closely associated with cell cycle-related molecules and pathways, such as Myc targets V1/2, DNA repair, mitotic spindle, G2M checkpoint and E2F targets. In addition, Peng et al (13) demonstrated that Zwint knockdown affected the expression of molecules associated with TNF signaling and other pathways. However, these potential molecular mechanisms of action of Zwint have not been confirmed by subsequent experiments. To the best of our knowledge, the present study was the first to demonstrate that Zwint promotes the malignant phenotype of melanoma by regulating c-Myc expression, which also supports previous findings with regards to the association between Zwint expression and the Myc pathway in bioinformatics analysis of gene expression profiles in breast cancer (10).

Dysregulation of the c-Myc oncogene has been observed in different types of tumor, such as liver and colon cancer, as well as Burkitt's lymphoma (21) and is known to contribute to the development of melanoma and other types of human cancer (22-24). The molecular mechanism regulating c-Myc expression is intricate. In addition to gene aberrances, such as genetic amplifications, insertions and translocations (25-27), a variety of factors can affect c-Myc expression. For example, Jiang et al (28) reported that TIP30 can repress estrogen receptor- $\alpha$-mediated c-Myc transcription. Furthermore, Sachdeva et al (29) demonstrated that microRNA-145 can inhibit c-Myc expression at the post-transcriptional level. c-Myc expression is also regulated by acetylation and ubiquitination (30-32). However, the specific molecular mechanism underlying Zwint regulation of c-Myc expression remains unclear and requires further study. Given that c-Myc is considered 'undruggable' at the protein level (33), Zwint, as a regulator of c-Myc, may be of value as a novel target for the treatment of melanoma.

In conclusion, the results of the present study demonstrated that Zwint expression was upregulated in melanoma and promoted tumor progression. Furthermore, depletion of Zwint was indicated to impair melanoma cell proliferation and migration, potentially by downregulating c-Myc expression. Taken together, these results provided novel insights into the molecular mechanism of melanoma development and suggested that Zwint may constitute a promising target for melanoma therapy.

\section{Acknowledgements}

Not applicable.

\section{Funding}

The present study was supported by the National Natural Science Foundation of China (grant no. 81802727) and the Fundamental Research Fund for Central Universities (grant no. xzy012019098).

\section{Availability of data and materials}

All data generated or analyzed during this study are included in this published article.

\section{Authors' contributions}

KM and RG conceived the experiments. KM, JZ and LW performed most of the experiments. KM and RG confirm the authenticity of all the raw data. XM and WL helped analyze the data. All authors discussed the results and RG drafted the initial manuscript. All authors have read and approved the final manuscript.

\section{Ethics approval and consent to participate}

Not applicable. 


\section{Patient consent for publication}

Not applicable.

\section{Competing interests}

The authors declare that they have no competing interests.

\section{References}

1. Coricovac D, Dehelean C, Moaca EA, Pinzaru I, Bratu T, Navolan D and Boruga O: Cutaneous melanoma-a long road from experimental models to clinical outcome: A review. Int J Mol Sci 19: 1566, 2018.

2. Siegel RL, Miller KD and Jemal A: Cancer statistics, 2020. CA Cancer J Clin 70: 7-30, 2020.

3. Rozeman EA, Dekker T, Haanen J and Blank CU: Advanced melanoma: Current treatment options, biomarkers, and future perspectives. Am J Clin Dermatol 19: 303-317, 2018.

4. Eggermont AM, Spatz A and Robert C: Cutaneous melanoma Lancet 383: 816-827, 2014

5. Woo SD, Yeop YS, Chung WJ, Cho DH, Kim JS and Su OJ: Zwint-1 is required for spindle assembly checkpoint function and kinetochore-microtubule attachment during oocyte meiosis. Sci Rep 5: 15431, 2015.

6. Starr DA, Saffery R, Li Z, Simpson AE, Choo KH, Yen TJ and Goldberg ML: HZwint-1, a novel human kinetochore component that interacts with HZW10. J Cell Sci 113: 1939-1950, 2000.

7. Vos LJ, Famulski JK and Chan GK: hZwint-1 bridges the inner and outer kinetochore: identification of the kinetochore localization domain and the hZw10-interaction domain. Biochem J 436: 157-168, 2011.

8. Famulski JK, Vos L, Sun X and Chan G: Stable hZW10 kinetochore residency, mediated by hZwint-1 interaction, is essential for the mitotic checkpoint. J Cell Biol 180: 507-520, 2008.

9. Faesen AC, Thanasoula M, Maffini S, Breit C, Müller F, van Gerwen S, Bange T and Musacchio A: Basis of catalytic assembly of the mitotic checkpoint complex. Nature 542: 498-502, 2017.

10. Li HN, Zheng WH, Du YY, Wang G, Dong ML, Yang ZF and Li XR: ZW10 interacting kinetochore protein may serve as a prognostic biomarker for human breast cancer: An integrated bioinformatics analysis. Oncol Lett 19: 2163-2174, 2020.

11. Ying $\mathrm{H}, \mathrm{Xu} \mathrm{Z}$, Chen $M$, Zhou S, Liang $X$ and Cai $X$ : Overexpression of Zwint predicts poor prognosis and promotes the proliferation of hepatocellular carcinoma by regulating cell-cycle-related proteins. Onco Targets Ther 11: 689-702, 2018

12. Xu Z, Zhou Y, Cao Y, Dinh TL, Wan J and Zhao M: Identification of candidate biomarkers and analysis of prognostic values in ovarian cancer by integrated bioinformatics analysis. Med Oncol 33: 130, 2016.

13. Peng F, Li Q, Niu SQ, Shen GP, Luo Y, Chen M and Bao Y: ZWINT is the next potential target for lung cancer therapy. J Cancer Res Clin Oncol 145: 661-673, 2019.

14. Kabbarah O, Nogueira C, Feng B, Nazarian RM, Bosenberg M, Wu M, Scott KL, Kwong LN, Xiao Y, Cordon-Cardo C, et al: Integrative genome comparison of primary and metastatic melanomas. PLoS One 5: e10770, 2010.

15. Livak KJ and Schmittgen TD: Analysis of relative gene expression data using real-time quantitative PCR and the 2(-Delta Delta C(T)) method. Methods 25: 402-408, 2001.

16. Ge R, Liu L, Dai W, Zhang W, Yang Y, Wang H, Shi Q, Guo S, Yi X, Wang G, et al: Xeroderma pigmentosum group A promotes autophagy to facilitate cisplatin resistance in melanoma cells through the activation of PARP1. J Invest Dermatol 136: $1219-1228,2016$
17. Zhou G, Shen M and Zhang Z: ZW10 binding factor (ZWINT), a direct target of Mir-204, predicts poor survival and promotes proliferation in breast cancer. Med Sci Monit 26: e921659, 2020.

18. Hoxhaj G and Manning BD: The PI3K-AKT network at the interface of oncogenic signalling and cancer metabolism. Nat Rev Cancer 20: 74-88, 2020

19. Cargnello M and Roux PP: Activation and function of the MAPKs and their substrates, the MAPK-activated protein kinases. Microbiol Mol Biol Rev 75: 50-83, 2011.

20. Multhaupt HA, Leitinger B, Gullberg D and Couchman JR: Extracellular matrix component signaling in cancer. Adv Drug Deliv Rev 97: 28-40, 2016.

21. Hsieh AL, Walton ZE, Altman BJ, Stine ZE and Dang CV: MYC and metabolism on the path to cancer. Semin Cell Dev Biol 43: 11-21, 2015

22. Dang CV: MYC on the path to cancer. Cell 149: 22-35, 2012.

23. Mannava S, Grachtchouk V, Wheeler LJ, Im M, Zhuang D, Slavina EG, Mathews CK, Shewach DS and Nikiforov MA: Direct role of nucleotide metabolism in C-MYC-dependent proliferation of melanoma cells. Cell Cycle 7: 2392-2400, 2008.

24. Zhuang D, Mannava S, Grachtchouk V, Tang WH, Patil S, Wawrzyniak JA, Berman AE, Giordano TJ, Prochownik EV, Soengas MS and Nikiforov MA: C-MYC overexpression is required for continuous suppression of oncogene-induced senescence in melanoma cells. Oncogene 27: 6623-6634, 2008.

25. Beroukhim R, Mermel CH, Porter D, Wei G, Raychaudhuri S, Donovan J, Barretina J, Boehm JS, Dobson J, Urashima M, et al: The landscape of somatic copy-number alteration across human cancers. Nature 463: 899-905, 2010

26. Niitsu N, Okamoto M, Miura I and Hirano M: Clinical features and prognosis of de novo diffuse large B-cell lymphoma with $\mathrm{t}(14 ; 18)$ and 8q24/c-MYC translocations. Leukemia 23: 777-783, 2009.

27. Rajan L, Broussard D, Lozano M, Lee CG, Kozak CA and Dudley JP: The c-myc locus is a common integration site in type B retrovirus-induced T-cell lymphomas. J Virol 74 : 2466-2471, 2000.

28. Jiang C, Ito M, Piening V, Bruck K, Roeder RG and Xiao H: TIP30 interacts with an estrogen receptor alpha-interacting coactivator CIA and regulates c-myc transcription. J Biol Chem 279: 27781-27789, 2004.

29. Sachdeva M, Zhu S, Wu F, Wu H, Walia V, Kumar S, Elble R, Watabe K and Mo YY: p53 represses c-Myc through induction of the tumor suppressor miR-145. Proc Natl Acad Sci U S A 106: 3207-3212, 2009

30. Wu H, Yang TY, Li Y, Ye WL, Liu F, He XS, Wang JR, Gan WJ, Li XM, Zhang S, et al: Tumor necrosis factor receptor-associated factor 6 promotes hepatocarcinogenesis by interacting with histone deacetylase 3 to enhance c-Myc gene expression and protein stability. Hepatology 71: 148-163, 2020.

31. Pan J, Deng Q, Jiang C, Wang X, Niu T, Li H, Chen T, Jin J, Pan W, Cai X, et al: USP37 directly deubiquitinates and stabilizes c-Myc in lung cancer. Oncogene 34: 3957-3967, 2015.

32. Patel JH, Du Y, Ard PG, Phillips C, Carella B, Chen CJ, Rakowski C, Chatterjee C, Lieberman PM, Lane WS, et al: The c-MYC oncoprotein is a substrate of the acetyltransferases hGCN5/PCAF and TIP60. Mol Cell Biol 24: 10826-10834, 2004.

33. Dang CV, Reddy EP, Shokat KM and Soucek L: Drugging the 'undruggable' cancer targets. Nat Rev Cancer 17: 502-508, 2017.

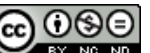

This work is licensed under a Creative Commons Attribution-NonCommercial-NoDerivatives 4.0 International (CC BY-NC-ND 4.0) License. 